\title{
Patient Characteristics and Outcomes Associated with Receiving an Earlier Versus Later Diagnosis of Probable Alzheimer's Disease
}

\author{
Noam Y. Kirson ${ }^{a}$, J. Scott Andrews ${ }^{\mathrm{b}}$, Urvi Desai ${ }^{\mathrm{a}, *}$, Sarah B. King ${ }^{\mathrm{a}}$, Sophie Schonfeld ${ }^{\mathrm{a}}$, \\ Howard G. Birnbaum ${ }^{\mathrm{a}}$, Daniel E. Ball ${ }^{\mathrm{b}}$ and Kristin Kahle-Wrobleski ${ }^{\mathrm{b}}$ \\ aAnalysis Group, Inc., Boston, MA, USA \\ ${ }^{\mathrm{b}}$ Eli Lilly and Company, Indianapolis, IN, USA
}

Accepted 6 September 2017

\begin{abstract}
.
Background: Effectiveness of Alzheimer's disease (AD) treatments may depend critically on the timeliness of intervention. Objective: To compare characteristics and outcomes of patients diagnosed with probable AD (prAD) based on time elapsed from first onset of cognitive decline.

Methods: Patients with $\geq 1$ prAD diagnosis and $\geq 1$ follow-up visit were selected from the National Alzheimer's Coordinating Center (NACC) Uniform Data Set (UDS; 9/2005-6/2015) and stratified based on the time between the perceived onset of cognitive decline at baseline and first prAD diagnosis (i.e., earlier versus later diagnosis). Characteristics at baseline and prAD diagnosis, clinically meaningful progression, and medication use following prAD diagnosis were compared.

Results: Median time from perceived onset of cognitive decline to prAD diagnosis was 4.5 years (earlier diagnosis: $\leq 3.46$; later diagnosis: $>5.71)$. Earlier-diagnosed patients $(n=1,476)$ were younger at baseline ( 74.3 versus 76.3 years) and had better cognitive and functional scores than later-diagnosed patients $(n=1,474)$. At first prAD diagnosis, earlier-diagnosed patients had lower mean global Clinical Dementia Rating (CDR) score (0.8 versus 1.1), higher mean Mini-Mental State Examination (MMSE) (22.6 versus 20.0), and lower mean Functional Activities Questionnaire (11.6 versus 17.3). Earlierand later-diagnosed patients experienced similar time to a decrease of $\geq 3$ points in MMSE (median 23.2 versus 23.1 months, $p=0.83$ ), but earlier-diagnosed patients had longer time to a CDR score of $\geq 2$ points, and longer times to initiation of $\mathrm{AD}$ medication and antipsychotic agents (all $p<0.01$ ).

Conclusion: Earlier prAD diagnosis in NACC data is associated with higher cognitive function and lower functional impairment at diagnosis.
\end{abstract}

Keywords: Alzheimer's disease, dementia, early diagnosis, longitudinal studies

\section{INTRODUCTION}

Alzheimer's disease (AD) is a slowly progressive neurodegenerative disease representing the most common cause of dementia [1]. In the US, it is

\footnotetext{
${ }^{*}$ Correspondence to: Urvi Desai, Analysis Group, Inc., 111 Huntington Avenue, 14th floor, Boston, MA 02199, USA. Tel.: +1 617425 8315; Fax: +1 617425 8001; E-mail: urvi.desai@analysis group.com.
}

estimated to affect as many as 5.2 million people aged 65 and older and 200,000 people under age 65 [1]. The neuropathological changes associated with $\mathrm{AD}$ begin to develop well before clinical symptoms emerge [2,3]. Indeed, patients are believed to experience a long pre-symptomatic (or pre-clinical) phase-lasting years or decades_-in which their cognitive ability remains normal despite the onset of the AD pathology [3-5]. This is followed by a 
prodromal (or pre-dementia) phase characterized by a progressive cognitive decline that typically starts with deficits in episodic memory and lasts several years $[3,4]$. As AD progresses and symptoms become more pronounced, the pre-dementia phase-also known as mild cognitive impairment (MCI) [3, 6]—eventually develops into overt dementia, defined as substantial impairments in both cognitive and functional domains $[5,7]$.

The diagnosis and management of patients with dementing illnesses can be challenging and uncertain as the underlying causes can be masked by many different factors, including side effects from medications and comorbidities such as depression and thyroid disorders [1,8]. Denial, fear, and lack of awareness and education among primary care physicians and caregivers have also been shown to hinder detection and diagnosis $[9,10]$. As a result, significant proportions of people with symptoms of cognitive impairment do not receive a diagnosis or receive a delayed diagnosis, when the symptoms become more apparent and easier to discern.

While several studies have described the projected economic implications of earlier diagnosis and treatment of $\mathrm{AD}$ [5, 11-13], little is known about the association between the timing of $\mathrm{AD}$ diagnosis and subsequent changes in clinical and functional outcomes. Having a better understanding of these outcomes is particularly important given that the effectiveness of future disease modifying treatments for AD may depend critically on the timeliness of intervention [11]. If started earlier in the disease continuum, treatment might be more likely to provide clinically relevant benefits and thus delay or prevent the onset of the highly disabling cognitive and functional impairments that are associated with later-stage $\mathrm{AD}[5]$.

To address this gap in the literature, the present study examined data for patients diagnosed with probable AD (prAD) at an Alzheimer's Disease Center (ADC). PrAD is defined by the National Institute of Neurological and Communicative Disorders and Stroke (NINCDS) and the Alzheimer's Disease and Related Disorders Association (ADRDA) criteria as the presence of cognitive deficits not attributable to other underlying conditions [14]. The study compared demographic and clinical characteristics at the time of a patient's initial visit to an ADC, as well as subsequent cognitive, functional, and treatment outcomes of prAD patients over time, stratified by the time that elapsed from the perceived onset of cognitive decline.

\section{MATERIALS AND METHODS}

\section{Data source}

The study used the publicly available National Alzheimer's Coordinating Center (NACC) Uniform Data Set (UDS), comprising data from the 34 past and present ADCs supported by the National Institute on Aging/National Institute of Health (NIA/NIH) [15]. The NACC, established in 1999, maintains a cumulative database including clinical evaluations, neuropathological data (when available) and MRI imaging (when available). The UDS reflects the total enrollment at the ADCs since September 2005 until June 2015, for a total of approximately 32,000 patients, and includes subjects with normal cognition, MCI, and dementia. Subjects may enroll in an $\mathrm{ADC}$, and therefore UDS, via clinician referral, self-referral, referral by family members, or active recruitment through community organizations; volunteers who wish to contribute to research on dementia may also enroll. Data for UDS are collected and recorded directly by trained clinicians and contain information on: 1) patient demographics, medical history (including medication use), and family history; 2) cognitive status, measured using validated instruments such as the Mini-Mental State Examination (MMSE) and Clinical Dementia Rating (CDR) scale; 3) functional status, evaluated using the Functional Assessment Questionnaire (FAQ); 4) behavioral symptoms, evaluated using the Neuropsychiatric Inventory Questionnaire (NPI-Q); and 5) clinical diagnoses of cognitive impairment.

\section{Sample selection}

The selection criteria used in this study are summarized in Fig. 1. The analytic sample was limited to patients with at least one record of a clinical diagnosis of prAD between September 2005 and June 2015 , corresponding to the most recent data available when data were requested. Patients in the study sample were required to have complete information regarding key demographic and clinical characteristics at the time of the initial visit to an ADC. In order to assess changes in cognitive and functional status over time, patients were also required to have at least one visit to an ADC after the first visit with a diagnosis of prAD and complete information about key measures of cognitive and functional status at their first visit with diagnosis of prAD as well as at the visit thereafter. 


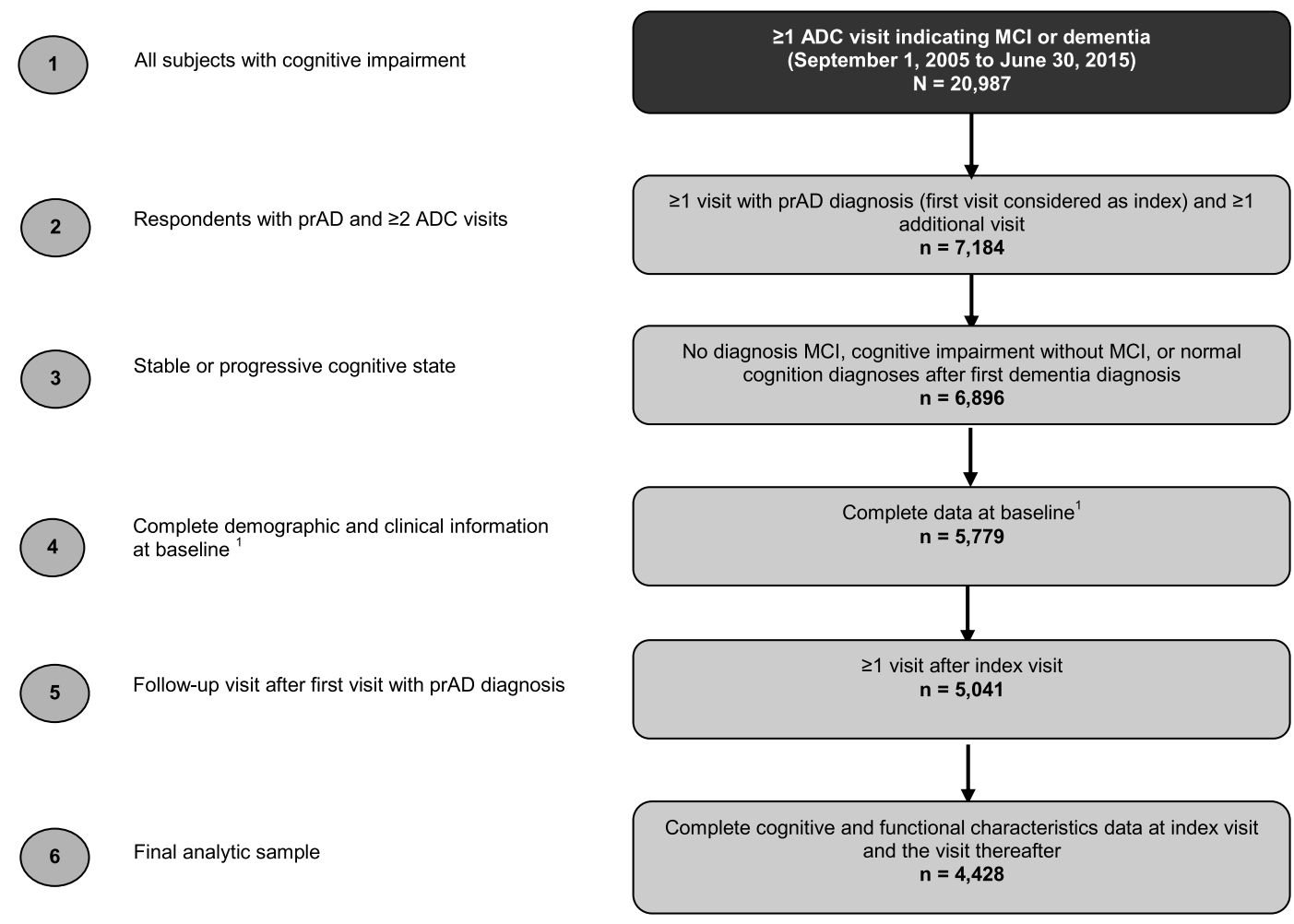

Fig. 1. Sample selection. ADC, Alzheimer's Disease Center; MCI, mild cognitive impairment; prAD, probable Alzheimer's disease. Note: Baseline was defined as the initial ADC visit.

Patients with improvement in cognitive status (defined as a diagnosis of MCI, cognitive impairment without MCI, or normal cognition) following the first visit with a diagnosis of prAD were excluded from the analysis.

\section{Study design}

The first visit to an ADC was defined as baseline visit. The first visit to an ADC with a diagnosis of prAD was defined as index visit. While patients could have multiple visits prior to their index visit, the baseline visit was the same as the index visit for $79 \%$ of patients. The follow-up period was the period after a diagnosis of prAD with $\geq 1$ and up to 10 visits to an ADC.

\section{Study cohorts}

Patients were stratified into two mutually exclusive cohorts based on the time between the perceived onset of cognitive decline and the first diagnosis of prAD recorded during an ADC visit (Fig. 2): patients in the bottom tertile (i.e., patients with the shortest time between the perceived onset of cognitive decline and the first diagnosis of prAD) were classified as having received an earlier diagnosis of prAD (earlier prAD cohort); patients in the top tertile (i.e., patients with the longest time between the perceived onset of cognitive decline and the first diagnosis of prAD) were classified as having received a later diagnosis of prAD (later prAD cohort).

\section{Age at perceived onset of cognitive impairment}

For most patients, the age at perceived onset of cognitive impairment was determined based on an NACC-derived variable that represents the age (as reported by patient, informant, or medical records) that cognitive decline began, ascertained during the baseline ADC interview. For the $1.3 \%$ of patients with missing or inconsistent values for this measure across visits, the age at cognitive decline onset was imputed based on the parameter estimates derived from a generalized linear model with the following predictors assessed at the baseline visit: demographics (age, gender, year of initial visit, race, and education); clinical characteristics (comorbid conditions 


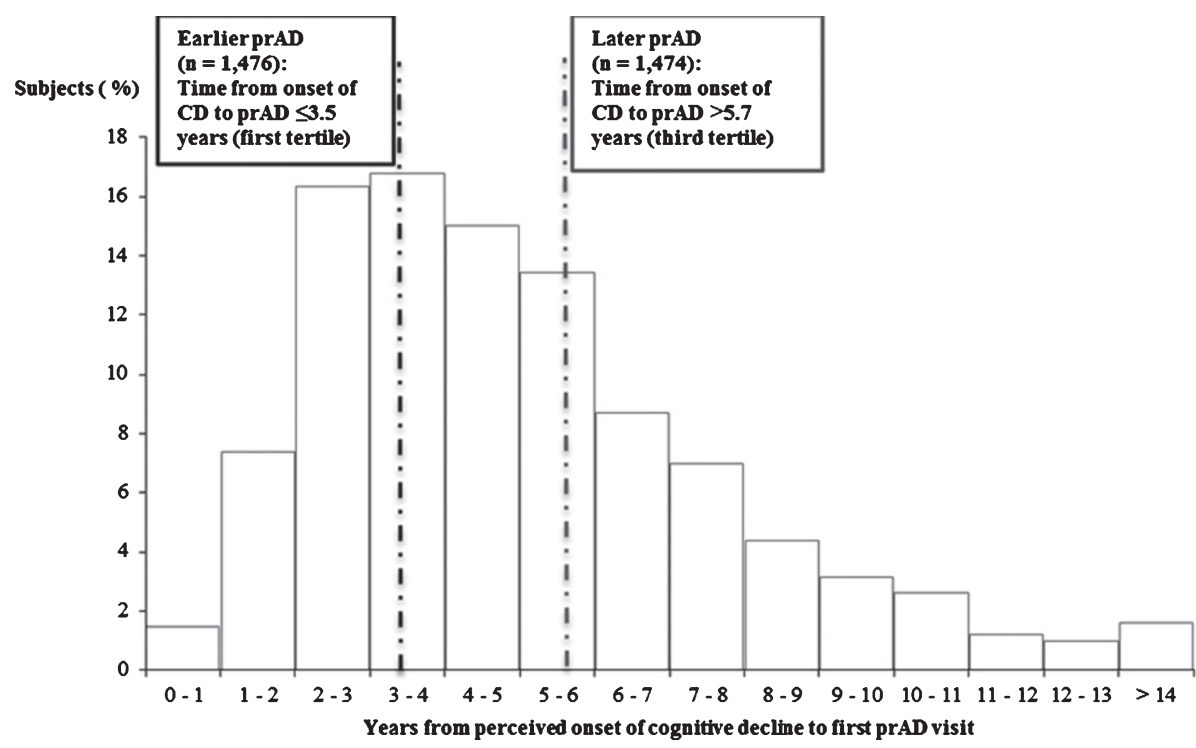

Fig. 2. Time from perceived onset of cognitive decline to prAD: earlier versus later prAD. prAD, probable Alzheimer's disease; $C D$, cognitive decline.

and number of APOE $\varepsilon 4$ alleles); cognitive characteristics (clinician diagnosis of cognitive status, consensus diagnosis, CDR Sum of Boxes and MMSE total score); and functional characteristics (FAQ score and level of independence).

\section{Patient characteristics}

The following patient characteristics were evaluated for the earlier and later prAD cohorts at baseline: demographics (age, gender, race, education, census division, living situation, and marital status); clinical characteristics (select comorbidities and APOE $\varepsilon 4$ allele status); cognitive characteristics (clinical diagnosis of cognitive status, mode of onset of symptoms, global CDR score, and MMSE score); functional and behavioral characteristics (FAQ score, self-reported level of independence, and NPI-Q score); and other characteristics, including year of initial visit, referral source, primary reason for ADC visit, and relationship of the informant with the patient.

The following characteristics were evaluated for the earlier and later prAD cohorts at the time of the index visit: cognitive characteristics (MMSE score, global CDR score); functional characteristics (FAQ score and proportion of patients not categorized as 'dependent', with dependence defined as scoring 3 points on $\geq 4$ items on the FAQ [16]); and medications (use of AD-related medications or antipsychotic agents).

\section{Study outcomes}

Outcomes were evaluated at each visit after the index visit. Cognitive and functional outcomes included: clinically meaningful decline in cognition, defined as a $\geq 3$ point decrease in MMSE total score [17, 18]; advancing stage of disease, defined as a global CDR score of $\geq 2$ [19]; and decline in functional status, defined as an increase of $\geq 1$ point on $\geq 4$ items on the FAQ [20]. Additional outcomes included receipt of AD-related medication (cholinesterase inhibitors or memantine) or antipsychotic agents.

\section{Statistical analyses}

Characteristics were compared between the earlier and later prAD cohorts using chi-square tests for proportions and Wilcoxon rank-sum tests for continuous variables.

Kaplan-Meier (K-M) survival analyses and multivariable Cox models were used to compare the time from the index visit to the development of outcomes between the two cohorts. Models adjusted for differences in the following metrics measured at baseline: 1) demographics (age, gender, race, and years of education); 2) year of initial visit; 3) primary referral source; 4) presence of psychiatric disorders; 5) cognitive characteristics - clinician diagnosis, global CDR score, mode of onset of cognitive symptoms (i.e., gradual [ $>6$ months], subacute $[\leq 6$ months], abrupt 
[within days], other, not applicable, or unknown); and 6) functional characteristics (FAQ score and level of independence).

\section{RESULTS}

In the study sample, the mean patient age at prAD diagnosis was approximately 75 years. The median time from perceived onset of cognitive impairment to prAD diagnosis was approximately 4.5 years $(4.3$ years for those diagnosed at baseline visit $[n=3,493]$ and 5.0 years for those diagnosed after baseline visit $[n=935])$.

Patients with a diagnosis of prAD within 3.5 years of cognitive impairment onset were assigned to the earlier prAD cohort $(n=1,476)$, whereas patients diagnosed after 5.7 years were assigned to the later prAD cohort $(n=1,474)$.

\section{Baseline characteristics}

Compared to patients in the later prAD cohort, patients in the earlier prAD cohort were significantly younger, less likely to be male, more likely to live alone, and less likely to have depression (Table 1). Patients in the earlier prAD cohort exhibited better cognitive functioning, as evidenced by higher MMSE scores and lower global CDR scores (Table 1). In addition, patients in the earlier prAD cohort were more likely to be either self-referred to the ADC or referred by relative/friend, less likely to require assistance with basic activities or be completely dependent, and were less likely to have neuropsychiatric symptoms, as demonstrated by a lower NPI-Q score (Table 1). Over $90 \%$ of the patients in both cohorts reported a gradual onset of symptoms of cognitive impairment (Table 1).

\section{Characteristics at the index visit}

Given that nearly $80 \%$ of the overall cohort had the same baseline and index visits, several clinical attributes of patients at the time of prAD diagnosis are similar to those reported at baseline. In particular, patients in the earlier prAD cohort had better cognitive and functional capabilities at the time of prAD diagnosis than patients in the later prAD cohort, as indicated by a significantly higher mean MMSE score and significantly lower global CDR score as well as FAQ score (Table 2). In addition, $92.3 \%$ of patients in the earlier prAD cohort were not categorized as dependent on the FAQ scale, compared to $72.3 \%$ of patients in the later prAD cohort $(p<0.05)$. In terms of medication use, significantly fewer patients in the earlier prAD cohort reported using AD-related medications or antipsychotics at the index visit compared to those in the later prAD cohort (Table 2).

\section{Time to changes in cognitive and functional outcomes}

The K-M-based median time from index visit to decrease of $\geq 3$ points in MMSE score was similar for the two cohorts (23.2 versus 23.1 months, Fig. 3; adjusted HR: 1.04, 95\% CI: [0.94, 1.15], Table 3). On the other hand, the K-M-based median time from the index visit to a score of $\geq 2$ points on the global CDR scale was significantly shorter for patients in the later prAD compared to those in the earlier prAD cohort (35.0 versus 40.7 months, $p<0.05$, Fig. 4); however, after adjusting for baseline differences, no difference was observed between the two cohorts (adjusted HR: $1.02,95 \%$ CI: [0.90, 1.17], Table 3).

The K-M-based median time from index visit to increase of $\geq 1$ point on $\geq 4$ items on the FAQ was significantly shorter for patients in the earlier prAD cohort compared to those in the later prAD cohort (17.7 versus 23.0 months, $p<0.05$, Fig. 5); however, after adjusting for baseline differences, no difference was observed between the two cohorts (adjusted HR: 1.07, 95\% CI: [0.96, 1.19]; Table 3).

\section{Time to initiating $A D$ medication or antipsychotics}

The K-M-based mean times to initiating ADrelated medications and antipsychotics were longer for patients in the earlier prAD cohort than for those in the later prAD cohort (AD medication: 8.4 versus 6.4 months, $p<0.05$; antipsychotics: 71.9 versus 67.0 months, $p<0.05$, Fig. 6A, B). After accounting for baseline characteristics, the difference in time to initiating AD-related medications persisted (adjusted HR: 0.91, 95\% CI: [0.83, 0.99]; Table 3) but the difference in time to initiating antipsychotics was no longer present (adjusted HR: 1.02; 95\% CI: [0.81, 1.28]; Table 3).

\section{DISCUSSION}

In this study, the mean age at perceived onset of cognitive impairment was approximately 70 years and the median time from onset of symptoms of cognitive decline to prAD diagnosis was over 4 years. 
Table 1

Baseline characteristics

\begin{tabular}{|c|c|c|c|}
\hline & $\begin{array}{c}\text { Earlier prAD } \\
(n=1,476)\end{array}$ & $\begin{array}{c}\text { Later prAD } \\
(n=1,474)\end{array}$ & $p$-value \\
\hline$\overline{\text { Age }(y), \text { mean (SD) }}$ & $74.3(9.9)$ & $76.3(8.9)$ & $<0.0001$ \\
\hline Male, $\%$ & 43.4 & 48.9 & 0.0028 \\
\hline Years of education, mean (SD) & $14.5(3.6)$ & $14.6(3.5)$ & 0.2592 \\
\hline Race,*\% & & & 0.0387 \\
\hline White/Caucasian & 82.5 & 85.9 & \\
\hline Black/African-American & 12.3 & 10.2 & \\
\hline Other & 5.2 & 3.9 & \\
\hline Living situation, $* \%$ & & & $<0.0001$ \\
\hline Lives alone & 17.8 & 12.1 & \\
\hline Lives with spouse/partner & 69.1 & 70.1 & \\
\hline Lives with relative/friend & 10.8 & 13.0 & \\
\hline Lives with group & 1.4 & 2.2 & \\
\hline Other & 1.0 & 2.6 & \\
\hline \multicolumn{4}{|l|}{ Select comorbidities, $\%$} \\
\hline Stroke & 4.5 & 5.6 & 0.1745 \\
\hline Depression & 38.6 & 42.7 & 0.0250 \\
\hline Alcohol abuse & 5.1 & 6.6 & 0.0822 \\
\hline Other psychiatric disorders & 4.5 & 5.5 & 0.2343 \\
\hline Number of APOE $\varepsilon 4$ alleles, $* \%$ & & & 0.5740 \\
\hline 0 & 33.3 & 31.5 & \\
\hline 1 & 37.1 & 38.1 & \\
\hline 2 & 11.8 & 13.1 & \\
\hline Unknown & 17.8 & 17.4 & \\
\hline Primary referral source, $* \%$ & & & $<0.0001$ \\
\hline Clinician & 48.9 & 52.5 & \\
\hline Self/relative/friend & 27.5 & 23.6 & \\
\hline ADC solicitation & 6.0 & 6.2 & \\
\hline Clinic sample & 2.4 & 5.4 & \\
\hline Non-ADC media appeal & 3.3 & 2.5 & \\
\hline Non-ADC study & 0.8 & 2.0 & \\
\hline Population sample & 1.1 & 0.5 & \\
\hline Other & 10.0 & 7.3 & \\
\hline Mode of onset of cognitive symptoms, $* \%$ & & & $<0.0001$ \\
\hline Gradual (>6 months) & 91.4 & 96.6 & \\
\hline Subacute $(<6$ months $)$ & 1.0 & 0.5 & \\
\hline Abrupt (within days) & 0.7 & 0.4 & \\
\hline Other & 0.1 & 0.1 & \\
\hline Not applicable & 6.0 & 2.0 & \\
\hline Unknown & 0.8 & 0.4 & \\
\hline Clinician diagnosis of cognitive status, $* \%$ & & & $<0.0001$ \\
\hline Normal cognition & 4.3 & 1.3 & \\
\hline Impaired, no MCI & 0.9 & 0.6 & \\
\hline MCI & 7.9 & 16.6 & \\
\hline Dementia & 86.9 & 81.5 & \\
\hline Global CDR score, mean (SD) & $0.8(0.4)$ & $1.1(0.7)$ & $<0.0001$ \\
\hline MMSE score, mean (SD) & 22.9 (4.4) & $20.8(6.1)$ & $<0.0001$ \\
\hline FAQ score, mean (SD) & $10.5(7.4)$ & $15.6(9.1)$ & $<0.0001$ \\
\hline Level of independence, $* \%$ & & & $<0.0001$ \\
\hline Able to live independently & 34.5 & 22.1 & \\
\hline Requires some assistance with complex activities & 53.8 & 46.3 & \\
\hline Requires some assistance with basic activities & 10.7 & 25.6 & \\
\hline Completely dependent & 1.0 & 6.0 & \\
\hline NPI-Q score, mean (SD) & $3.8(4.0)$ & $4.7(4.6)$ & $<0.0001$ \\
\hline
\end{tabular}

${ }^{*} p$-value reported for the joint significance test of difference in overall distribution. prAD, probable Alzheimer's disease; SD, standard deviation; APOE, apolipoprotein E; ADC, Alzheimer's Disease Center; MCI, mild cognitive impairment; CDR, Clinical Dementia Rating; MMSE, Mini-Mental State Examination; FAQ, Functional Assessment Questionnaire; NPI-Q, Neuropsychiatric Inventory Questionnaire. Note: $p$-values were estimated using chi-square tests for categorical variables and Wilcoxon rank-sum tests for continuous variables. 
Table 2

Characteristics at the time of the first prAD diagnosis

\begin{tabular}{lccc}
\hline & $\begin{array}{c}\text { Earlier prAD } \\
(n=1,476)\end{array}$ & $\begin{array}{c}\text { Later prAD } \\
(n=1,474)\end{array}$ & $p$-value \\
\hline MMSE score, mean (SD) & $22.6(4.3)$ & $20.0(5.9)$ & $<0.0001$ \\
Global CDR score, mean (SD) & $0.8(0.4)$ & $1.1(0.6)$ & $<0.0001$ \\
FAQ score, mean (SD) & $11.6(7.2)$ & $17.3(8.1)$ & $<0.0001$ \\
Patients not categorized as dependent on FAQ, $\%$ & 92.3 & 72.3 & $<0.0001$ \\
Medication use, $\%$ & & & \\
$\quad$ AD-related medications & 62.7 & 80.4 & $<0.0001$ \\
Antipsychotic agents & 2.6 & 6.2 & $<0.0001$ \\
\hline
\end{tabular}

prAD, probable Alzheimer's disease; MMSE, Mini-Mental State Examination; SD, standard deviation; CDR, Clinical Dementia Rating; FAQ, Functional Assessment Questionnaire; AD, Alzheimer's disease. Notes: Subjects with $\geq 4$ items at highest level (score of 3) were categorized as dependent on the FAQ. $p$-values were estimated using chi-square tests for categorical variables and Wilcoxon rank-sum tests for continuous variables.

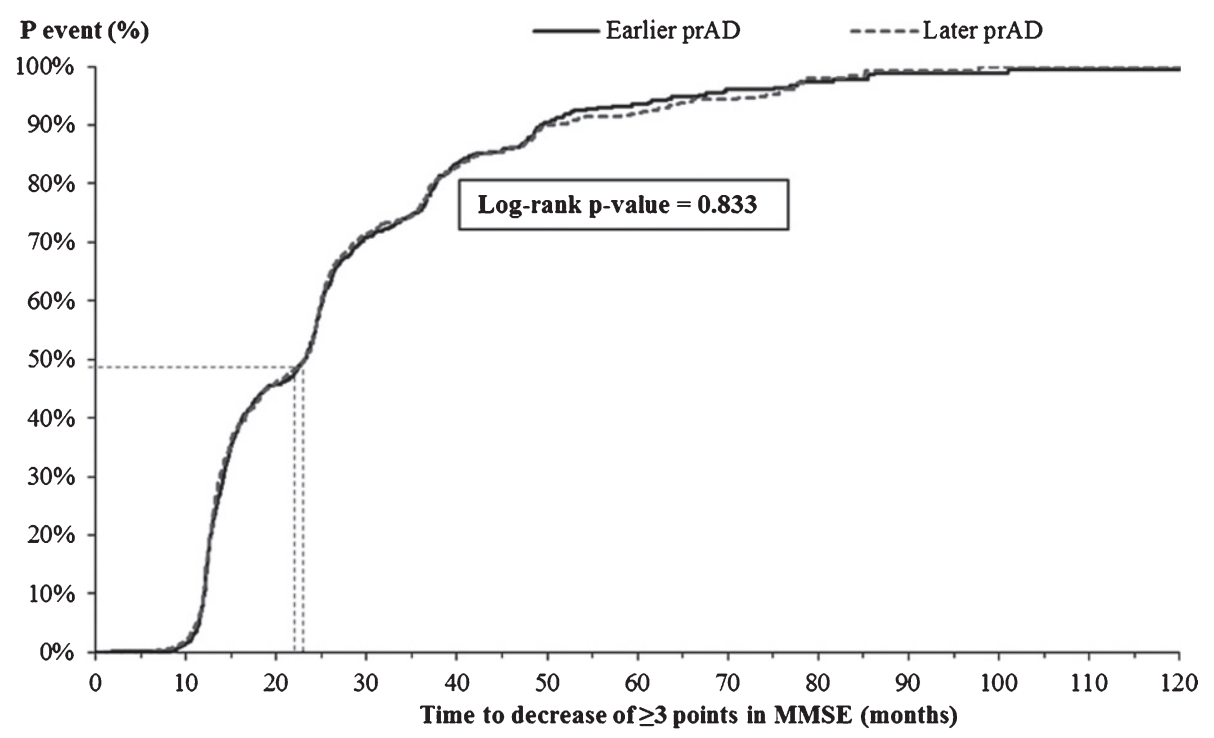

Fig. 3. Time from index visit to decrease of $\geq 3$ points in MMSE score. MMSE, Mini-Mental State Examination; p, probability; prAD, probable Alzheimer's disease. Note: Patients with an MMSE score $\leq 2$ at the first visit with prAD were censored at that visit. Otherwise, patients were censored at the last visit with complete information about MMSE.

Table 3

Likelihood of attaining outcomes among the Earlier prAD cohort relative to Later prAD cohort

\begin{tabular}{lcr}
\hline Outcome & Adjusted HR $(95 \% \mathrm{CI})$ & $p$-value \\
\hline Decrease of $\geq 3$ points in total MMSE score & $1.04(0.94,1.15)$ & 0.4555 \\
Reaching Global CDR Score $\geq 2$ & $1.02(0.90,1.17)$ & 0.7283 \\
Increase of $\geq 1$ points on $\geq 4$ items on the FAQ & $1.07(0.96,1.19)$ & 0.2222 \\
Initiating AD-related medications & $0.91(0.83,0.99)$ & 0.0272 \\
Initiating antipsychotics & $1.02(0.81,1.28)$ & 0.8822 \\
\hline
\end{tabular}

prAD, probable Alzheimer's disease; HR, hazard ratio; CI, confidence interval; MMSE, Mini-Mental State Examination; CDR, Clinical Dementia Rating; FAQ, Functional Assessment Questionnaire; AD, Alzheimer's disease. Notes: Models adjusted for differences in the following metrics measured at baseline: 1) demographics (age, gender, race, and years of education); 2) year of initial visit; 3) primary referral source; 4) presence of psychiatric disorders; 5) cognitive characteristics - clinician diagnosis, global CDR score, mode of onset of cognitive symptoms (i.e., gradual [ $>6$ months], subacute $[\leq 6$ months], abrupt [within days], other, not applicable, or unknown); and 6) functional characteristics (FAQ score and level of independence). For each outcome, patients were censored at the last visit with complete information about the outcomes of interest. 


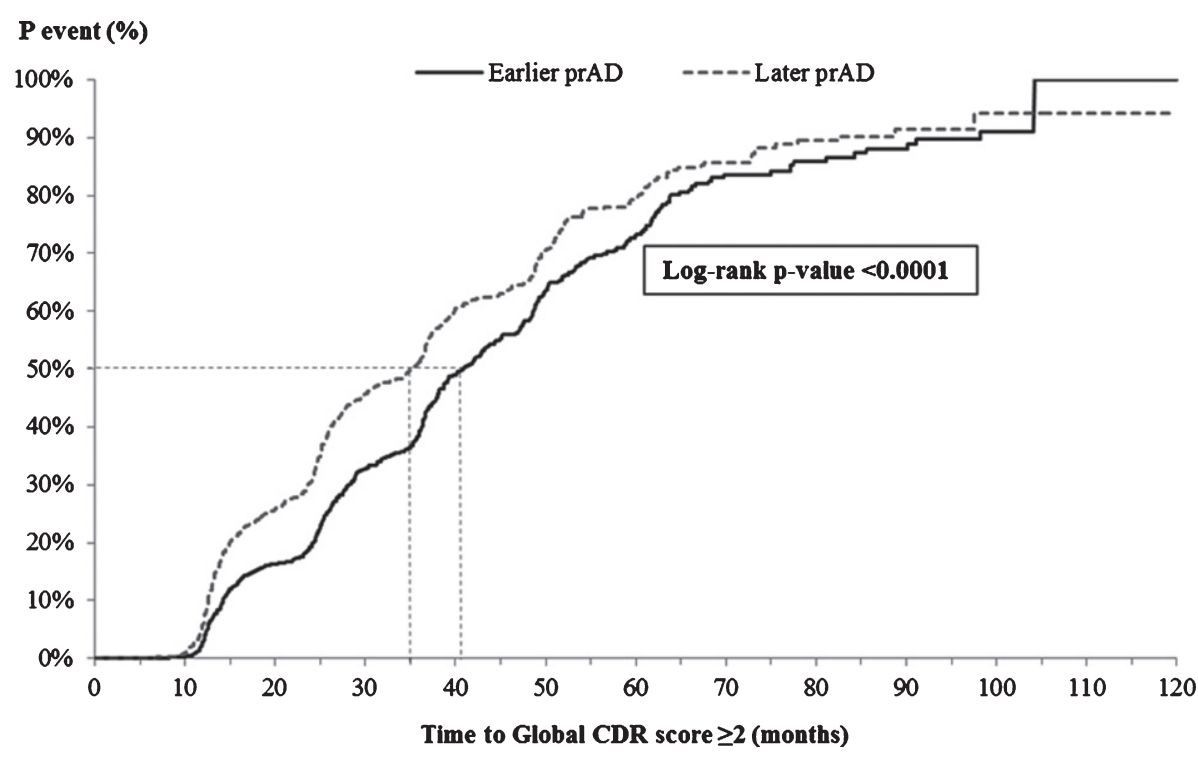

Fig. 4. Time from index visit to global CDR score $\geq 2$. CDR, Clinical Dementia Rating; $p$, probability; prAD, probable Alzheimer's disease. Note: Patients with a global CDR score of $\geq 2$ at the first visit with prAD were censored at that visit. Otherwise, patients were censored at the last visit with complete information about CDR.

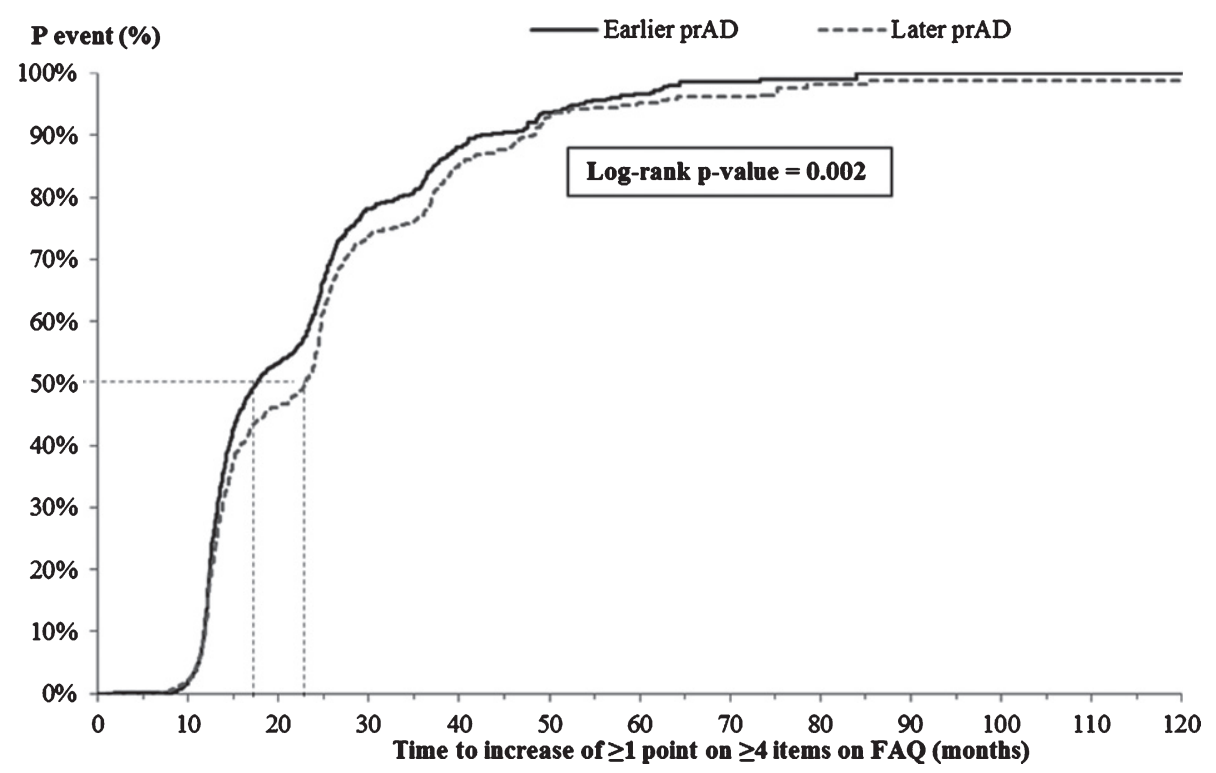

Fig. 5. Time from index visit to increase of $\geq 1$ point on $\geq 4$ items of the FAQ. FAQ, Functional Assessment Questionnaire; p, probability; prAD, probable Alzheimer's disease. Note: Patients without $\geq 4$ items on the FAQ with a severity $<3$ at the first visit with prAD were censored at that visit. Otherwise, patients were censored at the last visit with complete information about FAQ.

Almost $80 \%$ of the patients first presented to the ADC with symptoms consistent with prAD, suggesting that patients may not receive a diagnosis of prAD for extended periods of time after initial symptoms of cognitive decline appear.

When stratified by timing of prAD diagnosis relative to onset of symptoms, we found that patients receiving a diagnosis of prAD earlier (within 3.5 years of perceived onset of cognitive impairment) were relatively younger and had better cognitive performance (indicated by higher MMSE scores and lower global CDR scores), greater independence, greater functional capability (indicated by lower FAQ scores), and were more likely to live alone at diag- 


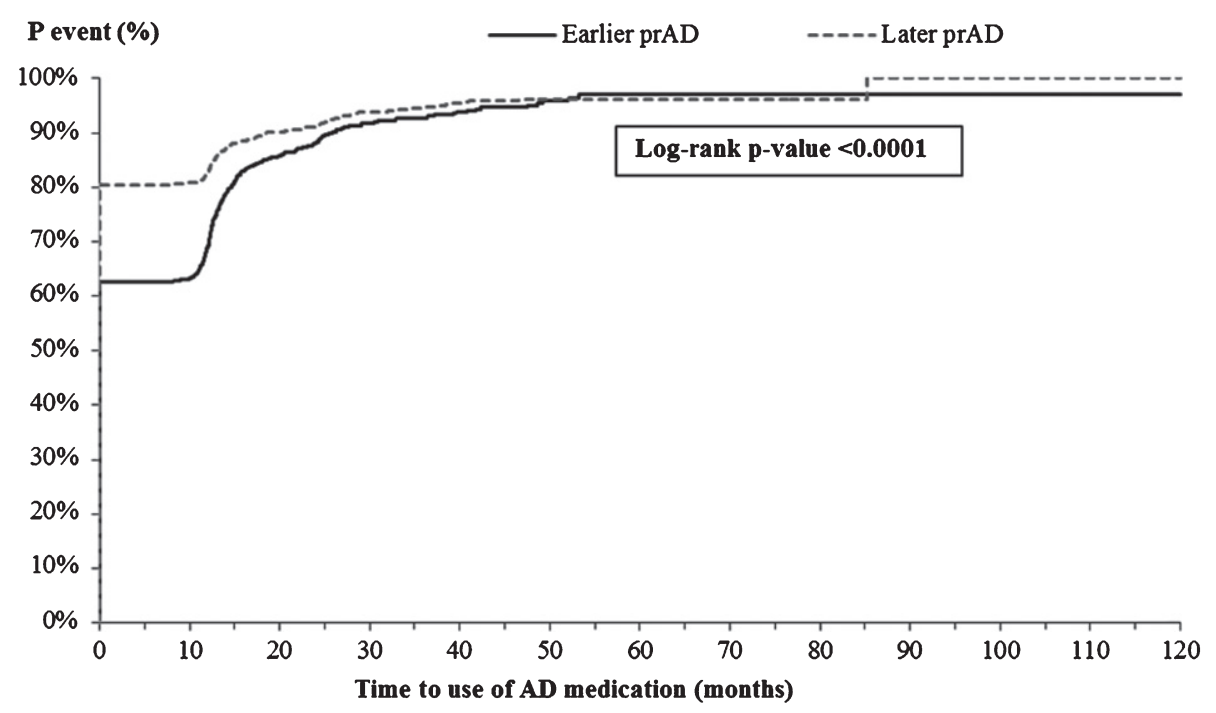

Fig. 6A. Time from index visit to initiating AD medication. AD, Alzheimer's disease; p, probability; prAD, probable Alzheimer's disease. Note: Patients were censored at the last visit with complete information about the use of antipsychotics.

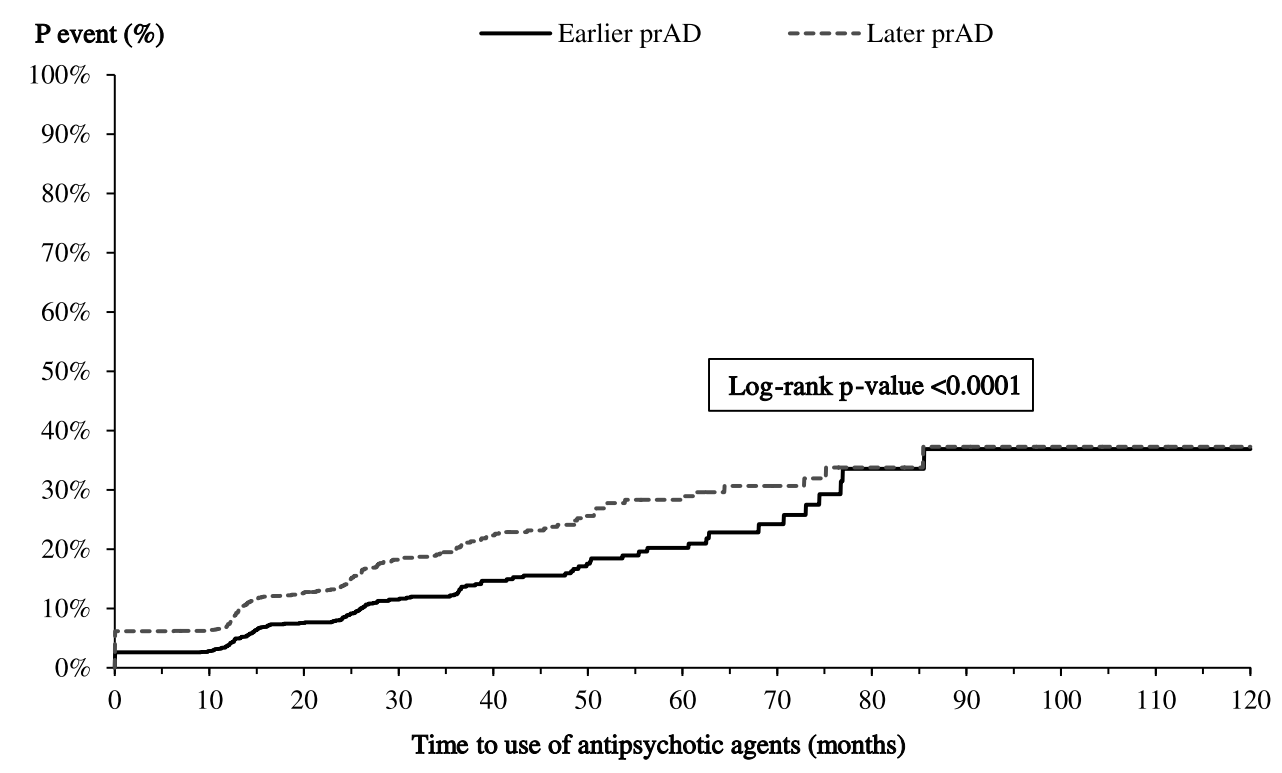

Fig. 6B. Time from index visit to initiating antipsychotics. p, probability; prAD, probable Alzheimer's disease. Note: Patients were censored at the last visit with complete information about the use of antipsychotics.

nosis compared with those diagnosed later (more than 5.7 years after onset of cognitive impairment). This data prompts some intriguing questions around why certain patients may get diagnosed sooner than others, particularly when their clinical symptoms appear to be milder. It is possible that those with an earlier diagnosis fit a more typical pattern of impairment/reported decline when evaluated during the clinical interview and initial work-up. Additional research on the types of symptoms reported, nature of decline, and results of neuropsychological testing could help answer this. The younger age, greater likelihood of living alone, and greater likelihood of being either self-referred to an ADC or referred to by relatives/friends suggests that perhaps the changes in memory were more salient or 
of greater importance to persons living alone and that seeking a diagnosis may in part be due to concerns about whether the person should remain living alone.

In terms of progression over time, a mixed picture emerged. Patients with an earlier prAD diagnosis displayed a similar time to a decrease of $\geq 3$ points in MMSE score as those with a later diagnosis. Coupled with a higher starting MMSE score, this implies relatively better cognitive abilities despite the decline. The longer time to a global CDR score $\geq 2$ among earlier diagnosed patients similarly implies a relative cognitive advantage (though both cohorts are clearly declining over time). In terms of functional decline, earlier diagnosed patients displayed a shorter time to an increase of $\geq 1$ point on $\geq 4$ items of the FAQ, suggesting that their progression is converging over time with later diagnosed patients. Many of these findings were no longer statistically significant after adjusting for baseline differences; however, this approach may be overly conservative as it controls for factors that may be inherently associated with the diagnosis timing.

The findings regarding severity at diagnosis along with the observed progression imply that earlier diagnosed patients likely have better clinical status for some period of time after their diagnosis relative to those diagnosed later. It is recognized that the longer time to reaching clinically meaningful thresholds for advancement of cognitive impairment in earlier diagnosed patients compared to later diagnosed patients may be correlated with the fact that those with an earlier prAD diagnosis have lower disease severity at diagnosis. Nonetheless, the study findings suggest that a greater benefit from future disease-modifying treatments may be received if people with symptoms consistent with an AD etiology are diagnosed and treated earlier, when their cognitive and functional capabilities are relatively intact. Indeed, using an economic model framework, Barnett et al. found that the effects of a disease modifying intervention that slows cognitive decline for 12 months after intervention would be greatest if the intervention were to be administered about 2 years prior to the standard diagnosis, when the cognitive capabilities began to deteriorate at a more pronounced rate [11]. In addition, emerging evidence (based on findings from clinical trials) suggests that the greatest opportunity to alter the course of AD may be to address the underlying disease process in the early stages of the disease [21-23]. This, coupled with the fact that, to date, no therapy with purported disease-modifying properties has demonstrated clinical benefit in advanced stages of the disease ( $C D R \geq 2$ ), further highlights the importance of earlier recognition and diagnosis of cognitive impairment due to AD. Additionally, the findings suggest that earlier diagnosis and detection of prAD may provide patients and their caregivers an opportunity for advanced planning as well as availing supportive services which can not only improve their quality of life, but also help reduce the overall care costs $[24,25]$.

In terms of medication use, we found that the earlier prAD cohort had significantly longer time to initiating AD-related medications as well as antipsychotic agents compared with the later prAD cohort. The difference in time to initiating AD-related medications persisted even after adjusting for baseline characteristics. These findings may be correlated with the fact that the earlier prAD cohort had lesser severity of disease at prAD diagnosis and, therefore, may not have been treated with medications. Considering that most antipsychotic medication use is deemed inappropriate for patients with dementia, a longer time to initiating these medications in the earlier prAD cohort suggests better care management for patients with prAD [26]. With regards to AD-related medications, however, several modeling studies have reported that even with existing symptomatic treatments, an earlier intervention can substantially improve patients' quality of life and reduce both economic and societal costs $[11,12]$. The magnitude of these benefits may be even higher should potential disease-modifying treatments be available in the future, as it may be possible to slow or stabilize cognitive and functional decline by diagnosing and treating patients earlier. For example, the study by Barnett et al. found that the maximum benefit of the disease-modifying intervention considered in their analysis was fifteen times greater than that for the symptomatic treatments [11].

\section{Study limitations}

The NACC UDS is a valuable resource for the AD research community because of the sample size and the wealth of clinical information it offers, and has been used in numerous publications. However, it is also important to note that for most (99\%) patients, the age at perceived onset of cognitive decline was identified using an NACC-derived variable, which, in turn is a measure based on the clinician's assessment 
of available medical records and patient's (or informant's) perception of the onset of cognitive issues, as opposed to an actual date of onset of symptoms, and therefore may be inaccurate. In addition, the overwhelming majority of the patients were diagnosed with prAD at the initial visit to the ADC; however, the precise date of potential $\mathrm{AD} /$ dementia diagnosis in other care settings was not available. Relatedly, the study was limited to patients with an established diagnosis of prAD and further research is needed to assess the disease progression trajectory among patients with milder forms of cognitive impairment (e.g., MCI). Furthermore, for the small subset of patients that did not receive a prAD diagnosis at first visit $(n=935)$, the median time from perceived onset of cognitive decline to diagnosis of prAD at an ADC visit was 5 years (versus 4.3 years for those with prAD diagnosis at first visit). Additional research is warranted to understand how the timing of diagnosis in relation to the onset of cognitive symptoms reported during ADC visits compares with general practice. Similarly, the medication use assessed in this study is self-reported (or informant-reported) and therefore should be interpreted with caution. In addition, while the study estimated the likelihoods of the various outcomes adjusted for differences in observable patient characteristics, the effects of unobservable heterogeneity across the two groups remain unknown. For example, some patients may seek medical advice earlier than others (either due to self-awareness or other factors such as accessibility to a provider), resulting in an earlier diagnosis. Finally, each ADC participating in the NACC UDS enrolls its subjects according to its own protocol. As a result, the study sample may not be representative of the broader population of patients with prAD or dementia. For example, NACC respondents have higher levels of education than the general US population.

\section{Conclusion}

In conclusion, earlier diagnosis of prAD in NACC data is associated with higher cognitive function, greater independence, and lower functional impairment at diagnosis. In terms of progression over time, both earlier and later prAD cohorts experienced declines in their cognitive capabilities; however, patients with an earlier prAD diagnosis continued to have relatively better cognitive abilities than those with a later diagnosis. The progression in functional capabilities for the two cohorts con- verged over time. These findings suggest that, as future disease-modifying therapies become available, earlier diagnosed patients could be treated while cognitive and functional abilities are greater, potentially increasing patient benefit. Additionally, a timely diagnosis provides an important opportunity for advanced planning and connecting patients and caregivers with supportive care services, as well as provides the opportunity to consider participation in clinical trials. Further research to better understand the patient, family, and clinician motivation for seeking a diagnosis sooner rather than later is also important, as this may inform how best to get appropriate care to patients in a timely manner.

\section{ACKNOWLEDGMENTS}

This study was sponsored by Eli Lilly and Company. J. Scott Andrews, Daniel E. Ball, and Kristin Kahle-Wrobleski are employees of Eli Lilly and Company and own stock. Noam Y. Kirson, Urvi Desai, and Howard G. Birnbaum are employees of Analysis Group Inc., which received research funding from Eli Lilly and Company for this study. Sarah B. King and Sophie Schonfeld were employees of Analysis Group, Inc. at the time of study. Medical writing services were provided by Cinzia Metallo, $\mathrm{PhD}$, an employee of Analysis Group, Inc., and were funded by Eli Lilly and Company. All the authors were involved in the study design, analyzed and interpreted the data, and contributed to manuscript development.

Authors' disclosures available online (http://j-alz. com/manuscript-disclosures/17-0078r3).

The NACC database is funded by NIA/NIH Grant U01 AG016976. NACC data are contributed by the NIA funded ADCs: P30 AG019610 (PI Eric Reiman, MD), P30 AG013846 (PI Neil Kowall, MD), P50 AG008702 (PI Scott Small, MD), P50 AG025688 (PI Allan Levey, MD, PhD), P50 AG047266 (PI Todd Golde, MD, PhD), P30 AG010133 (PI Andrew Saykin, PsyD), P50 AG005146 (PI Marilyn Albert, PhD), P50 AG005134 (PIBradley Hyman, MD, PhD), P50 AG016574 (PI Ronald Petersen, MD, PhD), P50 AG005138 (PI Mary Sano, PhD), P30 AG008051 (PI Steven Ferris, PhD), P30 AG013854 (PI M. Marsel Mesulam, MD), P30 AG008017 (PI Jeffrey Kaye, MD), P30 AG010161 (PI David Bennett, MD), P50 AG047366 (PI Victor Henderson, MD, MS), P30 AG010129(PICharles DeCarli, MD), P50 AG016573 
(PI Frank LaFerla, PhD), P50 AG016570 (PI MarieFrancoise Chesselet, MD, PhD), P50 AG005131 (PI Douglas Galasko, MD), P50 AG023501 (PI Bruce Miller, MD), P30 AG035982 (PI Russell Swerdlow, MD), P30 AG028383 (PI Linda Van Eldik, PhD), P30 AG010124 (PI John Trojanowski, MD, PhD), P50 AG005133 (PI Oscar Lopez, MD), P50 AG005142 (PI Helena Chui, MD), P30 AG012300 (PI Roger Rosenberg, MD), P50 AG005136 (PI Thomas Montine, MD, PhD), P50 AG033514 (PI Sanjay Asthana, MD, FRCP), P50 AG005681 (PI John Morris, MD), and P50 AG047270 (PI Stephen Strittmatter, MD, $\mathrm{PhD})$.

\section{PREVIOUS PRESENTATION}

A synopsis of the current research was presented in poster format at the Alzheimer's Association International Conference (AAIC), in Toronto, Canada, in July 2016.

\section{REFERENCES}

[1] Alzheimer's Association (2016) 2016 Alzheimer's disease facts and figures. Alzheimers Dement 12, 459-509.

[2] Mortimer JA, Borenstein AR, Gosche KM, Snowdon DA (2005) Very early detection of Alzheimer neuropathology and the role of brain reserve in modifying its clinical expression. J Geriatr Psychiatry Neurol 18, 218-223.

[3] Jack CR, Knopman DS, Jagust WJ, Shaw LM, Aisen PS, Weiner MW, Petersen RC, Trojanowski JQ (2010) Hypothetical model of dynamic biomarkers of the Alzheimer's pathological cascade. Lancet Neurol 9, 119.

[4] De-Paula VJ, Radanovic M, Diniz BS, Forlenza OV (2012) Alzheimer's disease. Subcell Biochem 65, 329-352.

[5] Dubois B, Padovani A, Scheltens P, Rossi A, Dell'Agnello G (2015) Timely diagnosis for Alzheimer's disease: A literature review on benefits and challenges. J Alzheimers Dis 49, 617-631.

[6] Roberts R, Knopman DS (2013) Classification and epidemiology of MCI. Clin Geriatr Med 29, 753-772.

[7] Burns A, Iliffe S (2009) Alzheimer's disease. BMJ 338, b158.

[8] Armstrong RA, Lantos PL, Cairns NJ (2005) Overlap between neurodegenerative disorders. Neuropathology 25, 111-124.

[9] Bond J, Stave C, Sganga A, O'Connell B, Stanley RL (2005) Inequalities in dementia care across Europe: Key findings of the Facing Dementia Survey. Int J Clin Pract Suppl 8-14.

[10] Bradford A, Kunik ME, Schulz P, Williams SP, Singh H (2009) Missed and delayed diagnosis of dementia in primary care: Prevalence and contributing factors. Alzheimer Dis Assoc Disord 23, 306-314.

[11] Barnett JH, Lewis L, Blackwell AD, Taylor M (2014) Early intervention in Alzheimer's disease: A health economic study of the effects of diagnostic timing. BMC Neurology 14, 1-9.
[12] Weimer DL, Sager MA (2009) Early identification and treatment of Alzheimer's disease: Social and fiscal outcomes. Alzheimers Dement 5, 215-226.

[13] Geldmacher DS, Kirson NY, Birnbaum HG, Eapen S, Kantor E, Cummings AK, Joish VN (2014) Implications of early treatment among Medicaid patients with Alzheimer's disease. Alzheimers Dement 10, 214-224.

[14] McKhann GM, Knopman DS, Chertkow H, Hyman BT, Jack CR Jr, Kawas CH, Klunk WE, Koroshetz WJ, Manly JJ, Mayeux R, Mohs RC, Morris JC, Rossor MN, Scheltens P, Carrillo MC, Thies B, Weintraub S, Phelps CH (2011) The diagnosis of dementia due to Alzheimer's disease: Recommendations from the National Institute on AgingAlzheimer's Association workgroups on diagnostic guidelines for Alzheimer's disease. Alzheimers Dement 7, 263-269.

[15] National Alzheimers Coordinating Center (NACC) (2016) Information and Resources, https://www.alz.washington. edu/WEB/researcher_home.html. Accessed August 2016.

[16] Chronic Care Networks for Alzheimer's Disease Initiative (2003) Tools for Early Identification, Assessment, and Treatment for People with Alzheimer's Disease and Dementia, https://www.alz.org/national/documents/bro chure_toolsforidassesstreat.pdf. Accessed September 2016.

[17] Clark CM, Sheppard L, Fillenbaum GG, Galasko D, Morris JC, Koss E, Mohs R, Heyman A (1999) Variability in annual Mini-Mental State Examination score in patients with probable Alzheimer disease: A clinical perspective of data from the Consortium to Establish a Registry for Alzheimer's Disease. Arch Neurol 56, 857-862.

[18] Burback D, Molnar FJ, St John P, Man-Son-Hing M (1999) Key methodological features of randomized controlled trials of Alzheimer's disease therapy. Minimal clinically important difference, sample size and trial duration. Dement Geriatr Cogn Disord 10, 534-540.

[19] Hughes CP, Berg L, Danziger WL, Coben LA, Martin RL (1982) A new clinical scale for the staging of dementia. $\mathrm{Br}$ J Psychiatry 140, 566-572.

[20] Lasser R, Soh C-H, Morrison G, Garibaldi G, Edgar C (2015) Identifying clinically relevant milestone decline in predementia Alzheimer's disease. Alzheimers Dement 11, P155-P156.

[21] Siemers ER, Sundell KL, Carlson C, Case M, Sethuraman G, Liu-Seifert H, Dowsett SA, Pontecorvo MJ, Dean RA, Demattos R (2016) Phase 3 solanezumab trials: Secondary outcomes in mild Alzheimer's disease patients. Alzheimers Dement 12, 110-120.

[22] Doody RS, Thomas RG, Farlow M, Iwatsubo T, Vellas B, Joffe S, Kieburtz K, Raman R, Sun X, Aisen PS, Siemers E, Liu-Seifert H, Mohs R (2014) Phase 3 trials of solanezumab for mild-to-moderate Alzheimer's disease. $N$ Engl J Med 370, 311-321.

[23] Salloway S, Sperling R, Fox NC, Blennow K, Klunk W, Raskind M, Sabbagh M, Honig LS, Porsteinsson AP, Ferris S, Reichert M, Ketter N, Nejadnik B, Guenzler V, Miloslavsky M, Wang D, Lu Y, Lull J, Tudor IC, Liu E, Grundman M, Yuen E, Black R, Brashear HR (2014) Two phase 3 trials of bapineuzumab in mildto-moderate Alzheimer's disease. $N$ Engl J Med 370, 322-333.

[24] Alzheimer's Association (2016) Medicare to provide coverage for critical care and support services for individuals living with Alzheimer's disease, http://alz.org/docu ments_custom/cms_release_110316.pdf. Accessed December 2016 
[25] Centers for Medicare and Medicaid Services (CMS) Medicare finalizes substantial improvements that focus on primary care, mental health, and diabetes prevention, https:// www.cms.gov/Newsroom/MediaReleaseDatabase/Press-re leases/2016-Press-releases-items/2016-11-02.html. Accessed December 2016.
[26] Banerjee S (2009) The use of antipsychotic medication for people with dementia: Time for action. A report for the Minister of State for Care Services, http://psychrights.org/re search/digest/nlps/BanerjeeReportOnGeriatricNeuroleptic Use.pdf. Accessed September 2016. 